\title{
Fully Hyperbolic Graph Convolution Network for Recommendation
}

\author{
Liping Wang ${ }^{1,2, *}$, Fenyu $\mathrm{Hu}^{1,2 *}$, Shu $\mathrm{Wu}^{1,2, \dagger}$, and Liang Wang ${ }^{1,2}$ \\ ${ }^{1}$ Center for Research on Intelligent Perception and Computing, Institute of Automation, Chinese Academy of Sciences \\ ${ }^{2}$ School of Artificial Intelligence, University of Chinese Academy of Sciences \\ wangliping2019@ia.ac.cn,fenyu.hu@cripac.ia.ac.cn \\ \{shu.wu,wangliang\}@nlpr.ia.ac.cn
}

\begin{abstract}
Recently, Graph Convolution Network (GCN) based methods have achieved outstanding performance for recommendation. These methods embed users and items in Euclidean space, and perform graph convolution on user-item interaction graphs. However, realworld datasets usually exhibit tree-like hierarchical structures, which make Euclidean space less effective in capturing user-item relationship. In contrast, hyperbolic space, as a continuous analogue of a tree-graph, provides a promising alternative. In this paper, we propose a fully hyperbolic GCN model for recommendation, where all operations are performed in hyperbolic space. Utilizing the advantage of hyperbolic space, our method is able to embed users/items with less distortion and capture user-item interaction relationship more accurately. Extensive experiments on public benchmark datasets show that our method outperforms both Euclidean and hyperbolic counterparts and requires far lower embedding dimensionality to achieve comparable performance.
\end{abstract}

\section{CCS CONCEPTS}

- Computer systems organization $\rightarrow$ Embedded systems; Redundancy; Robotics; • Networks $\rightarrow$ Network reliability.

\section{KEYWORDS}

recommendation system, graph neural networks, hyperbolic space

\section{ACM Reference Format:}

Liping Wang, Fenyu Hu, Shu Wu, and Liang Wang. 2018. Fully Hyperbolic Graph Convolution Network for Recommendation. In Woodstock '18: ACM Symposium on Neural Gaze Detection, June 03-05, 2018, Woodstock, NY. ACM, New York, NY, USA, 5 pages. https://doi.org/10.1145/1122445.1122456

\section{INTRODUCTION}

In information era, recommendation systems have been widely adopted to perform personalized information filtering [7, 25]. Even though there are many recommendation paradigms, collaborative

\footnotetext{
${ }^{*}$ The first two authors made equal contribution to this work.

${ }^{\dagger}$ To whom correspondence should be addressed.

Permission to make digital or hard copies of all or part of this work for personal or classroom use is granted without fee provided that copies are not made or distributed for profit or commercial advantage and that copies bear this notice and the full citation on the first page. Copyrights for components of this work owned by others than ACM must be honored. Abstracting with credit is permitted. To copy otherwise, or republish, to post on servers or to redistribute to lists, requires prior specific permission and/or a fee. Request permissions from permissions@acm.org.

Woodstock '18, fune 03-05, 2018, Woodstock, NY

(c) 2018 Association for Computing Machinery.

ACM ISBN 978-1-4503-XXXX-X/18/06 .. \$15.00

https://doi.org/10.1145/1122445.1122456
}

filtering $[8,24]$ which generates recommendations by utilizing available historical interactions, remains a fundamental and challenging task. The core idea behind collaborative filtering is to learn compact user/item embeddings and infer a user's preference to an item according to the distance between their embeddings.

From the perspective of graph, user-item interactions can be viewed as a bi-partite graph [3], where nodes represent users/items and edges represent their interactions. As a powerful tool of analyzing graph-structured data, Graph Neural Networks(GNNs) [9, $13,23]$ have recently demonstrated great success across various domains, including recommendation systems. Employing multiple layers of neighborhood aggregation, GNN-based methods [11, 24] have achieved the state-of-the-art performance on diverse public benchmarks.

Although GNN-based methods have achieved outstanding performance, it might not be appropriate to adopt Euclidean space to embed users and items. In real-world scenarios, user-item bipartite graphs usually exhibit tree-like hierarchical structures [2], in which the number of a node's neighbors grows exponentially with respect to the number of hops. Ideally, neighbors of node $v$ should be embedded in the ball centering at $v$ 's embedding, and the distance between embeddings should reflect the number of hops between nodes. Nevertheless, in Euclidean space, the volume of a ball only grows polynomially as a function of radius. Hence, embedding exponentially-growing number of neighbors into polynomiallygrowing size of volume would make distance between embeddings less accurate to reflect distance between nodes in the graph, and this is called distortion [6]. This kind of distortion makes it difficult to infer a user's preference to a target item according to the distance between their embeddings.

In contrast, hyperbolic space [6] in which the volume of a ball grows exponentially with radius offers a promising alternative. Compared with Euclidean space, hyperbolic space is more suitable for modeling user-item interaction graphs which exhibit strong tree-like hierarchical structures. Accordingly, it is a natural choice to conduct user/item embedding and graph convolution in hyperbolic space for recommendation. To the best of our knowledge, the only work adopting a similar idea is HGCF [19]. However, resorting to tangent space to realize graph convolution makes the performance of HGCF inferior for the two following reasons. On the one hand, tangent space is only local linear approximation of hyperbolic space. During the process of message propagation, errors caused by approximation accumulate and spread to the whole graph. As a result, influence from high-order neighbors cannot be captured accurately. On the other hand, tangent space is actually Euclidean 
space according to its definition [5]. Hence, the advantage of hyperbolic space in modeling user-item interaction relationship with less distortion cannot be fully utilized.

To overcome the limitation of Euclidean space and obtain more accurate user/item embeddings, we design a novel fully hyperbolic GCN framework specially for collaborative filtering. All operations are conducted in hyperbolic space, more specifically, in the Lorentz model, and we name it Lorentz Graph Collaborative Filtering (LGCF).

The main contributions of this work are summarized as follows:

- We propose a fully hyperbolic graph convolution network for recommendation.

- We conduct extensive experiments on multiple public benchmark datasets, and the results demonstrate the superiority of our method.

\section{PRELIMINARIES}

Problem Formulation. In this paper, the standard collaborative filtering set-up is considered. Let $U=\left\{u_{1}, u_{2}, \ldots, u_{n}\right\}$ be the set of $n$ users, and $I=\left\{i_{1}, i_{2}, \ldots, i_{m}\right\}$ be the set of $m$ items. Historical interactions between users and items are represented as a binary matrix $B$, where $B_{i, j}=1$ if the $i$-th user interacts with the $j$-th item, otherwise 0 . Given historical interactions $B$, the goal is to predict potential interactions.

GNN-based Recommendation Methods. GNN-based recommendation methods have received increasing attention for their ability to learn rich node representations. In collaborative filtering setting, GCN has replaced matrix factorization [17] and shown leading performance. In order to capture the influence from high-order neighbors, Wang et al. [24] proposed NGCF, a GCN framework performing on user-item interaction bi-partite graphs. Subsequently, He et al. [11] empirically found that two most common designs in GCNs - feature transformation and nonlinear activation, contribute little to the performance of collaborative filtering. Hence, they proposed a simplified architecture - LightGCN [11].

\section{OUR METHOD}

As illustrated in Figure 1, there are three components in LGCF: (1) an embedding layer that provides and initializes user/item embeddings in hyperbolic space; (2) multiple graph convolution layers that propagate user/item embeddings over the graph; and (3) a prediction layer that estimates a user's preference to an item by computing the distance between their embeddings.

\subsection{Embed Users/Items in Hyperbolic Space}

Existing GNN-based recommendation methods usually embed users and items in the same Euclidean space. To accurately model useritem interaction relationship, we investigate the utilization of hyperbolic space. There are several models of hyperbolic space, such as the Lorentz model, the Klein model and the Poincaré ball model. In this paper, we choose the Lorentz model due to its simplicity and numerical stability. Formally, the Lorentz model of $d$-dimensional hyperbolic space is defined as:

$$
\mathcal{L}=\left\{x=\left[x_{0}, x_{1}, \cdots, x_{d}\right] \in \mathbb{R}^{d+1}:\langle x, x\rangle_{\mathcal{L}}=-1, x_{0}>0\right\},
$$

where $\langle\boldsymbol{x}, \boldsymbol{y}\rangle_{\mathcal{L}}$ is Lorentz inner product and is defined as $\langle\boldsymbol{x}, \boldsymbol{y}\rangle_{\mathcal{L}}=$ $-x_{0} y_{0}+\sum_{i=1}^{d} x_{i} y_{i}$. In addition, at an arbitrary point $x \in \mathcal{L}$, hyperbolic space can be locally approximated by a linear Euclidean space. And this approximated Euclidean space is termed as tangent space $\mathcal{T}_{\boldsymbol{x}} \mathcal{L}$ in which norm $\|x\|_{\mathcal{L}}=\langle\boldsymbol{x}, \boldsymbol{y}\rangle$ is well defined. In LGCF, both users and items are embedded in the same Lorentz model of hyperbolic space.

It is well known that random initialization can have a significant impact on optimization in training [20]. A common practice in Euclidean space is Gaussian distribution initialization. Similarly, we design an initialization strategy for embeddings based on Wrapped Normal Distribution [15] which is a generalization of Gaussian distribution to hyperbolic space.

\subsection{Graph Convolution Layer}

The basic idea of GCN-based recommendation models is learning representations for users and items by aggregating neighbors' information iteratively over the interaction graph. In order to apply GCN for recommendation where users and items are embedded in hyperbolic space, we design graph convolution layers specially since naïve generalization will drive embeddings out of hyperbolic space. Before that, we give a brief review of existing graph convolution layers in Euclidean space.

In Euclidean space, a graph convolution operation is composed of three steps: feature transformation, neighborhood aggregation and non-linear activation. Among them, feature transformation is performed through linear transformation. In LGCF, we discard the feature transformation operation for two reasons. On the one hand, different from attributed graphs (e.g., citation networks) where nodes bring rich feature information, nodes in user-item interaction graphs contain no semantics but one-hot IDs. In this case, feature transformation may provide no benefits, and could bring difficulties to training. On the other hand, linear transformation (matrix-vector multiplication) is not well-defined in hyperbolic space since it is not a vector space.

Neighborhood Aggregation. Exising neighborhood aggregation of the $l$-th layer can be summarized as:

$$
\boldsymbol{e}_{i}^{(l)}=\sum_{j \in \hat{\mathcal{N}}(i)} w_{i j} \boldsymbol{e}_{j}^{(l-1)},
$$

in which $\boldsymbol{e}_{j}^{(l-1)}$ represents the $j$-th node's embedding and $\hat{\mathcal{N}}(i)$ denotes the set consisting of node $i$ and its neighborhood nodes. A natural generalization of mean to hyperbolic space is Einstein midpoint [22]. However, it is defined in the Klein model $\mathcal{K}=$ $\left\{x \in \mathbb{R}^{d}:\|x\|<1\right\}$, while user/item embeddings lie in the Lorentz model. Luckily, there are isometric maps between the Klein model and the Lorentz model defined as follows:

$$
\begin{aligned}
F(x) & =\frac{\left[x_{1}, x_{2}, \ldots x_{d}\right]}{x_{0}}, \\
F^{-1}(\boldsymbol{k}) & =\frac{1}{1-\|\boldsymbol{k}\|^{2}}[1, \boldsymbol{k}],
\end{aligned}
$$

in which $x=\left[x_{0}, x_{1}, \ldots, x_{d}\right] \in \mathcal{L}$ and $\boldsymbol{k} \in \mathcal{K}$.

Hence, we propose a neighborhood aggregation strategy utilizing the Klein model as an intermediate bridge. Specifically, neighborhood aggregation can be divided into three steps. First, current 


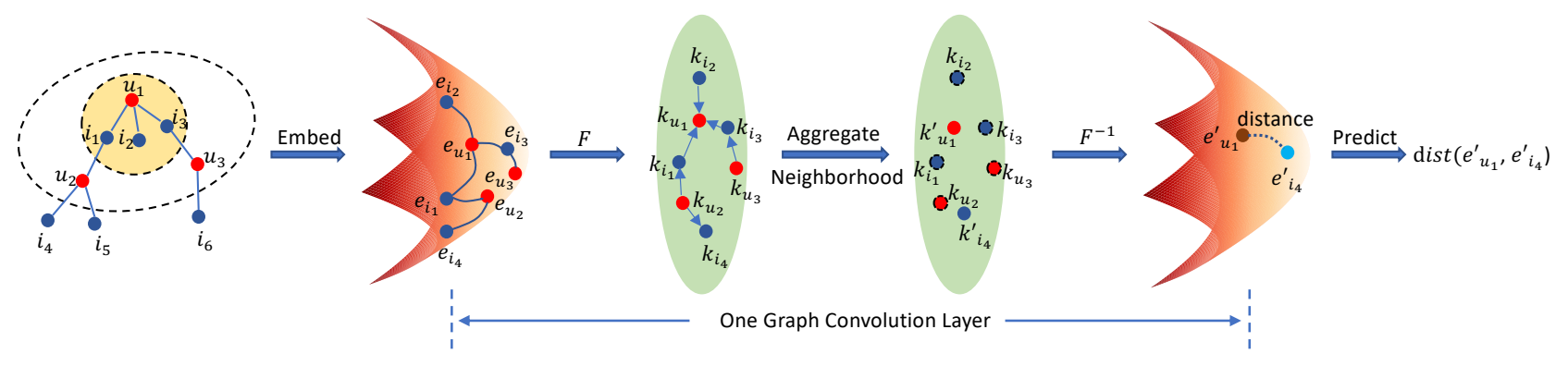

Figure 1: Illustration of LGCF. First, users and items are embedded into the Lorentz model of hyperbolic space. Then, multiple graph convolution layers (only one shown in the figure for simplicity) are adopted to aggregate information from neighbors. In each layer, embeddings are first mapped by $F$ to the Klein model in which graph convolution is performed. After that, $F^{-1}$ maps refined embeddings back to the Lorentz model. Finally, LGCF infers a user's preference to an item according to the distance between their embeddings.

embeddings $\left\{\boldsymbol{e}_{1}, \boldsymbol{e}_{2}, \ldots, \boldsymbol{e}_{n}\right\}$ are mapped from the Lorentz model to the Klein model by $\boldsymbol{k}_{i}=F\left(\boldsymbol{e}_{i}\right)$. Then, neighborhood aggregation is conducted in the Klein model as follows:

$$
\begin{array}{r}
\gamma_{i}=\frac{1}{\sqrt{1-\left\|k_{j}\right\|^{2}}}, \\
\boldsymbol{k}_{i}^{\prime}=\frac{\sum_{j \in \hat{N}(i)} \gamma_{j} \boldsymbol{k}_{j}}{\sum_{j \in \hat{N}(i)} \gamma_{j}} .
\end{array}
$$

Here, we obtain aggregated user/item embeddings in the Klein model. Last, aggregated embeddings $\boldsymbol{k}_{i}^{\prime}$ are mapped back to the Lorentz model through $z_{i}=F^{-1}\left(\boldsymbol{k}_{i}^{\prime}\right)$.

Nonlinear Activation Layer. In Euclidean space, nonlinear activation has proven to be a key component in modern neural networks. However, direct adoption will drive the computation result out of hyperbolic space. To fix this problem, we design a calibration strategy following general activation. Formally, let $x_{i}=$ $\left[x_{0}, x_{1}, \ldots, x_{d}\right]=\sigma\left(z_{i}\right)$ be the output of general activation function $\sigma$, e.g., ReLU. The first element of $x$ is calibrated while the other elements remain unchanged to pull the activated embedding back to hyperbolic space:

$$
\boldsymbol{e}_{i}^{\prime}=f_{c}\left(x_{i}\right)=\left[\sqrt{1+\sum_{j=1}^{d} x_{j}^{2}}, x_{1}, x_{2}, \ldots, x_{d}\right] .
$$

\subsection{Prediction Layer}

After propagating with $L$ graph convolution layers, we obtain multiple representations for users and items. Representations generated in different layers emphasize messages passed through different connections, and they reflect users' preference or items' attributes from different perspectives. Recommendation models operating in hyperbolic space usually estimate a user's preference to a target item according to the distance or similarity metric between their representations. In the Lorentz model, the generalization of a straight line in Euclidean space is geodesics which gives the shortest distance between two points $\boldsymbol{x}, \boldsymbol{y}$. Formally, the geodesics distance between $x$ and $y$ is defined as:

$$
d_{\mathcal{L}}(x, y)=\operatorname{arcosh}\left(-\langle x, \boldsymbol{y}\rangle_{\mathcal{L}}\right)
$$

Hence, in LGCF, we infer the preference of user $u$ to item $i$ based on the geodesics distance between their corresponding representations. Further, to utilize different semantics captured by different layers, we take representations learned by different layers into consideration simultaneously. In summary, LGCF estimates the preference of user $u$ to target item $i$ as:

$$
y(u, i)=\frac{1}{\sum_{l=1}^{L} d_{\mathcal{L}}^{2}\left(\boldsymbol{e}_{u}^{(l)}, \boldsymbol{e}_{i}^{(l)}\right)},
$$

in which $\boldsymbol{e}_{u}^{(l)}$ and $\boldsymbol{e}_{i}^{(l)}$ are representations generated by the $l$-th layer. Even though there are multiple choices for layer aggregation, such as weighted average, max pooling, LSTM, etc., we find simple summation adopted here works well empirically.

\subsection{Margin Ranking Loss}

Margin ranking loss [21] has been a competitive choice for distancebased recommendation systems. Since it encourages positive and negative user-item pairs to be separated up to a given margin. Once the difference between a negative user-item pair and a positive one is greater than the margin, these two user-item pairs will make no contribution to the loss. In this way, hard pairs violating the margin are focused all the time, making optimization much easier. We extend margin ranking loss to hyperbolic space based on geodesics distance. Given a sampled positive user-item pair $(u, i)$ and a negative one $(u, j)$, geodesics margin loss is defined as:

$$
\ell_{g}(u, i, j)=\sum_{l=1}^{L} \min \left(d_{\mathcal{L}}^{2}\left(\boldsymbol{e}_{u}^{(l)}, \boldsymbol{e}_{j}^{(l)}\right)-d_{\mathcal{L}}^{2}\left(\boldsymbol{e}_{u}^{(l)}, \boldsymbol{e}_{i}^{(l)}\right)-m, 0\right),
$$

where $m$ is a non-negative hyper-parameter. Note that representations obtained by different layers contribute to the loss simultaneously. This not only makes it possible to utilize different semantics captured by different layers, but also decreases the difficulty of optimization due to the residual connection [10].

\subsection{Optimization}

The only parameter of LGCF is the embedding matrix of users and items. These embeddings lie in the Lorentz model of hyperbolic space which is out of the range of common optimization algorithms 
Table 1: Dataset statistics.

\begin{tabular}{l|ccc}
\hline Dataset & \#User & \#Item & \#Interactions \\
\hline Amazon-CD & 22,947 & 18,395 & 422,301 \\
Amazon-Book & 52,406 & 41,264 & $1,861,118$ \\
Yelp2020 & 91,174 & 45,063 & $1,940,014$ \\
\hline
\end{tabular}

Table 2: Recall results for all datasets.

\begin{tabular}{l|c|c|cc|cc|c}
\hline Datasets & Metrics & BPRMF & NGCF & LightGCN & HVAE & HGCF & LGCF \\
\hline \multirow{2}{*}{ Amazon-CD } & R@10 & 0.0779 & 0.0758 & 0.0929 & 0.0781 & $\underline{0.0962}$ & $\mathbf{0 . 0 9 9 6}$ \\
& R@20 & 0.1200 & 0.1150 & 0.1404 & 0.1147 & $\underline{0.1455}$ & $\mathbf{0 . 1 5 0 3}$ \\
\hline \multirow{2}{*}{ Amazon-Book } & R@10 & 0.0611 & 0.0658 & 0.0799 & 0.0774 & $\underline{0.0867}$ & $\mathbf{0 . 0 8 9 9}$ \\
& R@20 & 0.0794 & 0.1050 & 0.1248 & 0.1125 & $\underline{0.1318}$ & $\mathbf{0 . 1 3 6 0}$ \\
\hline \multirow{2}{*}{ Yelp2020 } & R@10 & 0.0325 & 0.0458 & 0.0522 & 0.0421 & $\underline{0.0543}$ & $\mathbf{0 . 0 5 7 3}$ \\
& R@20 & 0.0556 & 0.0764 & 0.0866 & 0.0691 & $\underline{0.0884}$ & $\mathbf{0 . 0 9 4 6}$ \\
\hline
\end{tabular}

Table 3: NDCG results for all datasets.

\begin{tabular}{l|c|c|cc|cc|c}
\hline Datasets & Metrics & BPRMF & NGCF & LightGCN & HVAE & HGCF & LGCF \\
\hline \multirow{2}{*}{ Amazon-CD } & N@10 & 0.0610 & 0.0591 & 0.0726 & 0.0629 & $\underline{0.0751}$ & $\mathbf{0 . 0 7 8 0}$ \\
& N@20 & 0.0974 & 0.0718 & 0.0881 & 0.0749 & $\underline{0.0909}$ & $\mathbf{0 . 0 9 4 5}$ \\
\hline \multirow{2}{*}{ Amazon-Book } & N@10 & 0.0594 & 0.0655 & 0.0780 & 0.0778 & $\underline{0.0869}$ & $\mathbf{0 . 0 9 0 6}$ \\
& N@20 & 0.0971 & 0.0791 & 0.0938 & 0.0901 & $\underline{0.1022}$ & $\mathbf{0 . 1 0 6 3}$ \\
\hline \multirow{2}{*}{ Yelp2020 } & N@10 & 0.0283 & 0.0405 & $\underline{0.0461}$ & 0.0371 & 0.0458 & $\mathbf{0 . 0 4 8 5}$ \\
& N@20 & 0.0512 & 0.0513 & 0.0582 & 0.0465 & $\underline{0.0585}$ & $\mathbf{0 . 0 6 1 2}$ \\
\hline
\end{tabular}

such as SGD [18] and Adam [12]. Hence, we employ RGSD [4], a generation of SGD to hyperbolic space, which mimics SGD's behavior while taking into account the geometry of hyperbolic space.

\section{EXPERIMENTS}

\subsection{Set Up}

Datasets and Baselines. Following HGCF [19], we employ AmazonCD [16], Amazon-Book [16] and Yelp2020 [1] datasets. Dataset statistics are provided in Table 1. Each dataset is split into 80-20 train and test sets. Multiple competitive baseline methods from three categories are compared: BPRMF [17], NGCF [24], LightGCN [11], HVAE [14], HGCF [19]. Among them, BPRMF optimizes matrix factorization by Bayesian personalize ranking (BPR) loss [17]. NGCF and LightGCN employ GNN in Euclidean space. HVAE combines variational auto-encoder(VAE) with hyperbolic geometry. Last, HGCF applies the latest Hyperbolic GCN [6] to recommendation systems.

Implementation. For a fair comparison, the embedding dimensionality is set to 50 for all methods, and the same negative sampling strategy is adopted. For all baseline methods, suggested settings in original papers are followed. Grid search for hyper-parameters are conducted following HGCF [19]. For LGCF, the number of GCN layers is set to 3 . We set the learning rate to 0.001 and weight decay to 0.005 . And model is trained for 1000 epochs. Hyper-parameter margin $m$ is tuned from $[0.01,2]$.
Table 4: Results for LGCF and LGCF-tangent on Yelp2020.

\begin{tabular}{l|cccc}
\hline & R@10 & R@20 & N@10 & N@20 \\
\hline LGCF & 0.0573 & 0.0946 & 0.0485 & 0.0612 \\
LGCF-tangent & 0.0545 & 0.0895 & 0.0463 & 0.0586 \\
\hline
\end{tabular}
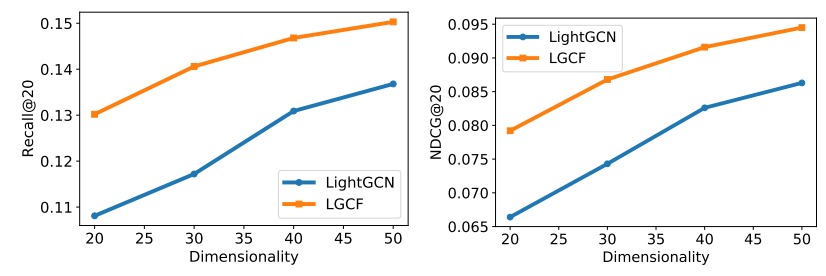

Figure 2: Recall@20 and NDCG@20 on Amazon-CD dataset with an embedding dimensionality varying from 20 to 50 .

\subsection{Overall Results}

Recall and NDCG results for all datasets are reported in Table 2 and Table 3 respectively. We can see that LGCF consistently outperforms other methods on all the three datasets. Compared with LightGCN, LGCF achieves an improvement up to $\mathbf{1 6 . 1 5 \%}$ in NDCG@10 on Amazon-Book dataset, demonstrating the superiority of hyperbolic space over Euclidean space in modeling real-world user-item interactions.

Among the baseline methods, HGCF is the most competitive counterpart of LGCF. Even though HGCF adopts hyperbolic space, it resorts to tangent space to conduct aggregation operations, which brings inevitable distortion. In contrast, LGCF performs all graph convolution operations in hyperbolic space. LGCF outperforms HGCF with wide margins on all the datasets, showing the information loss introduced by tangent space in HGCF.

\subsection{Ablation Study}

To further analyze the effect of fully hyperbolic graph convolution network, we conduct an ablation study on Yelp2020, the largest one among the three datasets. Since simply replacing fully hyperbolic graph convolution with regular Euclidean graph convolution would drive user/item embeddings out of hyperbolic space, we conduct convolution operations in tangent space, and name this model variant as LGCF-tangent. From experimental results shown in Table 4, we can observe that there is a wide margin between the performance of LGCF and LGCF-tangent. This is because, in LGCF-tangent, errors caused by tangent space approximation accumulate and spread to the whole graph. As a result, influence from neighbors, especially high-order neighbors, cannot be captured accurately.

\subsection{Effect of Embedding Dimensionality}

In order to validate the advantage of hyperbolic space to learn compact representations, we compare the results of LGCF and LightGCN with different values of embedding dimensionality. From Figure 2, we can observe that LGCF outperforms LightGCN consistently at 
all dimensionality values, and the greatest margin occurs at lower dimensionality. LGCF requires far lower embedding dimensionality to achieve comparable performance to its Euclidean analogue. This reflects that LGCF's advantage is more prominent when the embedding dimensionality cannot be large due to limited computing and storage resources.

\section{CONCLUSION}

In this paper, we propose LGCF, a fully hyperbolic GCN model for recommendation. Utilizing the advantage of hyperbolic space, LGCF is able to embed users/items with less distortion and capture user-item interaction relationship more accurately. Extensive experiments on public benchmark datasets show that LGCF outperforms both Euclidean and hyperbolic counterparts and requires far lower embedding dimensionality to achieve comparable performance.

\section{REFERENCES}

[1] 2020. Yelp Open Dataset. https://www.yelp.com/dataset

[2] Aaron B Adcock, Blair D Sullivan, and Michael W Mahoney. 2013. Tree-like structure in large social and information networks. In IEEE International Conference on Data Mining.

[3] Béla Bollobás. 2013. Modern graph theory.

[4] Silvere Bonnabel. 2013. Stochastic gradient descent on Riemannian manifolds. IEEE Trans. Automat. Control (2013).

[5] William M Boothby. 1986. An introduction to differentiable manifolds and Riemannian geometry.

[6] Ines Chami, Rex Ying, Christopher Ré, and Jure Leskovec. 2019. Hyperbolic graph convolutional neural networks. Advances in Neural Information Processing Systems (2019)

[7] Paul Covington, Jay Adams, and Emre Sargin. 2016. Deep neural networks for youtube recommendations. In Proceedings of the 10th ACM Conference on Recommender Systems.

[8] Travis Ebesu, Bin Shen, and Yi Fang. 2018. Collaborative memory network for recommendation systems. In The 41st International ACM SIGIR Conference on Research \& Development in Information Retrieval.

[9] William L. Hamilton, Rex Ying, and Jure Leskovec. 2017. Inductive Representation Learning on Large Graphs. In Advances in Neural Information Processing Systems.

[10] Kaiming He, Xiangyu Zhang, Shaoqing Ren, and Jian Sun. 2016. Deep Residual Learning for Image Recognition. In IEEE Conference on Computer Vision and Pattern Recognition.

[11] Xiangnan He, Kuan Deng, Xiang Wang, Yan Li, Yongdong Zhang, and Meng Wang. 2020. Lightgen: Simplifying and powering graph convolution network for recommendation. In Proceedings of the 43rd International ACM SIGIR Conference on Research and Development in Information Retrieval.

[12] Diederik P Kingma and Jimmy Ba. 2014. Adam: A method for stochastic optimization. arXiv preprint arXiv:1412.6980 (2014)

[13] Thomas N. Kipf and Max Welling. 2017. Semi-Supervised Classification with Graph Convolutional Networks. In International Conference on Learning Representations.

[14] Leyla Mirvakhabova, Evgeny Frolov, Valentin Khrulkov, Ivan Oseledets, and Alexander Tuzhilin. 2020. Performance of Hyperbolic Geometry Models on Top-N Recommendation Tasks. In Fourteenth ACM Conference on Recommender Systems.

[15] Yoshihiro Nagano, Shoichiro Yamaguchi, Yasuhiro Fujita, and Masanori Koyama. 2019. A wrapped normal distribution on hyperbolic space for gradient-based learning. In International Conference on Machine Learning.

[16] Jianmo Ni, Jiacheng Li, and Julian McAuley. 2019. Justifying recommendations using distantly-labeled reviews and fine-grained aspects. In Proceedings of the 2019 Conference on Empirical Methods in Natural Language Processing and the 9th International foint Conference on Natural Language Processing.

[17] Steffen Rendle, Christoph Freudenthaler, Zeno Gantner, and Lars Schmidt-Thieme. 2009. BPR: Bayesian Personalized Ranking from Implicit Feedback. In Proceedings of the Twenty-Fifth Conference on Uncertainty in Artificial Intelligence.

[18] Herbert Robbins and Sutton Monro. 1951. A stochastic approximation method. The Annals of Mathematical Statistics (1951).

[19] Jianing Sun, Zhaoyue Cheng, Saba Zuberi, Felipe Pérez, and Maksims Volkovs. 2021. HGCF: Hyperbolic Graph Convolution Networks for Collaborative Filtering. (2021).

[20] Ilya Sutskever, James Martens, George Dahl, and Geoffrey Hinton. 2013. On the importance of initialization and momentum in deep learning. In International Conference on Machine Learning.

[21] Yi Tay, Luu Anh Tuan, and Siu Cheung Hui. 2018. Latent relational metric learning via memory-based attention for collaborative ranking. In Proceedings of the 2018 World Wide Web Conference.

[22] Abraham Albert Ungar. 2008. A gyrovector space approach to hyperbolic geometry. Synthesis Lectures on Mathematics and Statistics (2008).

[23] Petar Veličković, Guillem Cucurull, Arantxa Casanova, Adriana Romero, Pietro Liò, and Yoshua Bengio. 2018. Graph Attention Networks. International Conference on Learning Representations (2018).

[24] Xiang Wang, Xiangnan He, Meng Wang, Fuli Feng, and Tat-Seng Chua. 2019. Neural graph collaborative filtering. In Proceedings of the 42nd international ACM SIGIR Conference on Research and Development in Information Retrieval.

[25] Rex Ying, Ruining He, Kaifeng Chen, Pong Eksombatchai, William L Hamilton, and Jure Leskovec. 2018. Graph convolutional neural networks for web-scale recommender systems. In Proceedings of the 24th ACM SIGKDD International Conference on Knowledge Discovery \& Data Mining. 